\title{
Mitspieler aktivieren
}

\section{Behandlung bei Belastungsinkontinenz Durch Prostata-OPs}

ist Beckenbodentraining auch für viele Männer relevant. Ein

Programm fern von Kirschkernpicken und Aufzugfahren ist entscheidend. Denn mit diesen Übungen lässt sich Mann selten motivieren und - viel wichtiger - weder die Beckenbodenmuskulatur noch ihre Synergisten adäquat trainieren.

Die Zahl der radikalen Prostataektomien (RPE) ist in den letzten Jahrzehnten - infolge der Entdeckung des prostataspezifischen Antigens (PSA) - massiv gestiegen. Eine schwedische Studie spricht von einer 25-fachen Steigerung zwischen 1988 und 2008 [1]. Die Ärzte stellen die OP-Indikation für eine RPE seit ein paar Jahren zwar wieder deutlich vorsichtiger, dennoch lag 2012 die OP-Rate in Deutschland bei rund 25.000 [2]. Und auch wenn die OP-Techniken mittlerweile schonender sind, leiden zwischen 3 und 69 Prozent der Männer post-OP an einer Belastungsinkontinenz. Die Zahlen variieren stark, je nachdem wie Kontinenz definiert wurde und ob Patient oder Operateur befragt wurde [3-5].

Klassisches Beckenbodentraining ist unzureichend $\rightarrow$ Physiotherapie ist bei Belastungsinkontinenz unverzichtbar. Das unterstreicht das Leitlinienprogramm Onkologie in seiner S3-Leitlinie zur Früherkennung, Diagnose und Therapie der verschiedenen Stadien des Prostatakarzinoms, die Physiotherapie mit dem Empfehlungsgrad A und dem Evidenzlevel 1+ nennt: „Im Mittelpunkt des Kontinenztrainings bei Belastungsinkontinenz soll die Physiotherapie stehen“ [6]. Die Basis „klassischer“ Physiotherapieprogramme bilden meist Spannungsübungen des Beckenbodens und der urethralen Sphinktermuskulatur, die der Gynäkologe Arnold H. Kegel erstmals in den 1950er Jahren empfohlen hat. Doch abgesehen davon, dass sich vor allem Männer mit Kirschkernpicken und Aufzugfahren schwertun, lässt sich damit die Beckenbodenmuskulatur (BBM) in vielen Fällen nicht ausreichend trainieren - weder die weibliche noch die männliche.

Rumpf- und Beinbereich beeinflussen den Beckenboden $\rightarrow$ Grundsätzlich sollte man die anatomischen Zusammenhänge immer umfassend betrachten und Rumpf- und Beinbereich einbeziehen. Denn der 
in verschiedene Stränge eingeteilte M. levator ani ist größtenteils nicht direkt mit den Beckenknochen verbunden, sondern über einen sehnig-faszialen Bogen unter anderem mit dem M. obturatorius internus, dem stärksten Außenrotator des Hüftgelenks. Zudem steht der Beckenboden zum Beispiel mit dem M. iliopsoas, den Mm. transversus und rectus abdominis sowie den Glutäen in Beziehung. Strukturen, die so miteinander verbunden sind, beeinflussen sich häufig gegenseitig. Ist der M. obturatorius internus beispielsweise geschwächt oder in seiner Elastizität eingeschränkt, was bei Patienten in der Altersgruppe 60+ oft der Fall ist, ist es entscheidend, diesen auch zu behandeln. Die Funktionen und Strukturen im Rumpf- und Beinbereich sollte der Therapeut daher unbedingt in Befund und Behandlung einbeziehen ( $\odot$ „DER ZAPPLER“, S. 46). Bei Patienten mit einer Langzeitinkontinenz sind diese Bereiche häufig sogar der Knackpunkt, und ein isoliertes Training von M. levator ani und M. sphincter externus mit den Kegel-Übungen ist unzureichend. Im Falle größerer Gewebeschäden der Schließmuskulatur kann zudem eine gut funktionierende Rumpf- und Beinmuskulatur zur Kompensation beitragen.

Beckenbodenmuskulatur am besten im Verbund trainieren $\rightarrow$ 2008 zeigte Ruth Sapsford in Australien an einer Gruppe von Physiotherapiestudentinnen, dass die BBM von selbst aktiviert wird, wenn die Probandinnen aufrecht saßen, das Becken kippten und den Brustkorb hoben [7]. In dieser Position waren fast 25 Prozent der maximal möglichen BBM-Spannung nachweisbar, in entspannter, angelehnter Haltung nur circa sieben Prozent. Haltungsschulung ist daher beim Beckenbodentraining zentral (॰ „DER KONZERTPIANIST“, S. 46). Die Grundspannung im aufrechten Sitz ist ein idealer Reiz, der sich oft und über lange Zeit im Tagesablauf integrieren lässt.

Zusammen mit Zwerchfell, Bauch- und Rückenmuskulatur ist das Diaphragma pelvis an der Aktivität des Körpers gegen die Schwerkraft beteiligt. Da dies aber nur im Verbund bewerkstelligt werden kann, können Dysbalancen wie Tonusdifferenzen dazu führen, dass der Synergismus in seiner Funktion gestört ist. Versucht man in diese subkortikal organisierten Abläufe einzugreifen, verschlechtert man das Gesamtergebnis, statt es zu optimieren. Deutlich wird das, wenn man beispielsweise seine Sprunghöhe mit und ohne eingezogenen Bauch vergleicht. Mit Bauchspannung ist das Ergebnis deutlich schlechter. Daher ist es für das Training komplexer Aufgaben nicht sinnvoll, Muskelgruppen einzeln anspannen zu lassen, zum Beispiel die BBM isoliert zu aktivieren, um die Kontinenz für das Aufstehen zu trainieren. Empfehlenswert ist es, den Beckenboden, das Zwerchfell, die Bauchund Rückenmuskulatur im Verbund zu trainieren ( $\odot$ „BANKSTELLUNG“, „WALKING PLANKING“ UND „ÜBERALL MACHBAR“, S. 46-47).

In zahlreichen Untersuchungen hat sich gezeigt, dass der M. levator ani synergistisch auf die Bauchmuskelaktivität reagiert, und zwar proportional zur Druckgröße, die intraabdominal entsteht [8-14]. Darüber hinaus lassen Forschungsergebnisse von Paul Hodges und Kollegen von 2007 vermuten, dass die BBM sogar schon vor den anderen posturalen Muskeln aktiv sein könnte [14]. Und auch die Studien von Helena Luginbuehl und Kollegen scheinen zu belegen, dass es spontane „Feed-forward-Schleifen“ gibt und die BBM physiologisch schon Millisekunden vor der Belastungssituation aktiv ist [15]. Es handelt sich also offensichtlich um eine neuromus-

\section{i physiokongress 2017 Beckenbodentraining}

Auf dem physiokongress vom 27. bis 29.1.2017 spricht Markus Martin über das Thema „Kontinent nach Prostataoperation“. Er stellt sein ganzheitliches Behandlungskonzept „BM Balance“ vor, das Dysfunktionen im Bereich Becken/Blase/Prostata vorbeugt und beseitigt und den Beckenboden in seinem natürlichen Synergismus fordert.

kuläre Steuerung, die „unterhalb“ des motorischen Kortex anzusiedeln ist. Diese isoliert zu trainieren, ist kaum möglich. Vielmehr ist es entscheidend, den geforderten Regelkreis in entsprechenden Situationen zu bahnen ( $\odot$ „DER BAUCHBÄNDIGER“, S. 47).

Bei intraabdominalem Druck senkt sich der Blasenhals physiologisch $\rightarrow$ Was unter anderem Kavita Singh bereits 2002 bei ValsalvaManövern (forciertes Ausatmen bei verschlossener Mund- und Nasenöffnung) im MRT nachweisen konnte [16], zeigten Janis Miller und Kollegen in einer Untersuchung von 2005 [17]: Der Blasenhals senkt sich beim Husten normalerweise ab - und zwar sowohl bei inkontinenten als auch bei kontinenten Frauen. Die Absenkung des M. levator ani während intraabdominaler Druckerhöhung ist also eher physiologisch als pathologisch einzustufen. Die BBM muss sich in dieser Situation anspannen und gleichzeitig verlängern, sprich zu exzentrischer Muskeltätigkeit fähig sein. Diese Leistungsfähigkeit lässt sich einzig mit exzentrischem Training verbessern, isometrisch und konzentrisches Training reichen nicht aus.

Erfreulicherweise ergänzen oder modifizieren viele BBM-Rehabilitationskonzepte inzwischen die Kegel-Übungen. Was bislang aber oft fehlt, sind individuell orientierte Maßnahmen, die auch mal überhaupt keine Kegel-Übungen verlangen. Ziel muss es sein, die Patienten in ihrer Gesamtkompetenz zu einem möglichst physiologischen Verhalten zurückzubringen und eine automatisierte Beckenbodenaktivität zu erreichen. Hierfür braucht es ein individuell konzipiertes Vorgehen, das die genannten anatomischen und physiologischen Faktoren ebenso berücksichtigt wie die persönlichen Aspekte wie Ausdauer, Bewegungsverhalten und mentale Kompetenz. Markus Martin

$\Rightarrow$ Literaturverzeichnis und Patienteninformation zum Download www.thieme-connect.de/products/physiopraxis > "Ausgabe 10/16“

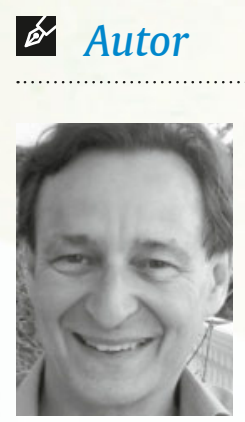

Markus Martin ist Physiotherapeut sowie Ausbilder für Physiotherapie, Urologie und Gynäkologie. Seine therapeutischen Spezialgebiete sind Beschwerden rund um Blase, Prostata und Beckenboden beim Mann. Er berät seine Patienten bei erektiler Dysfunktion und zum Thema Prostataoperationen. 


\section{So trainieren Sie Ihre Beckenbodenmuskulatur optimal}

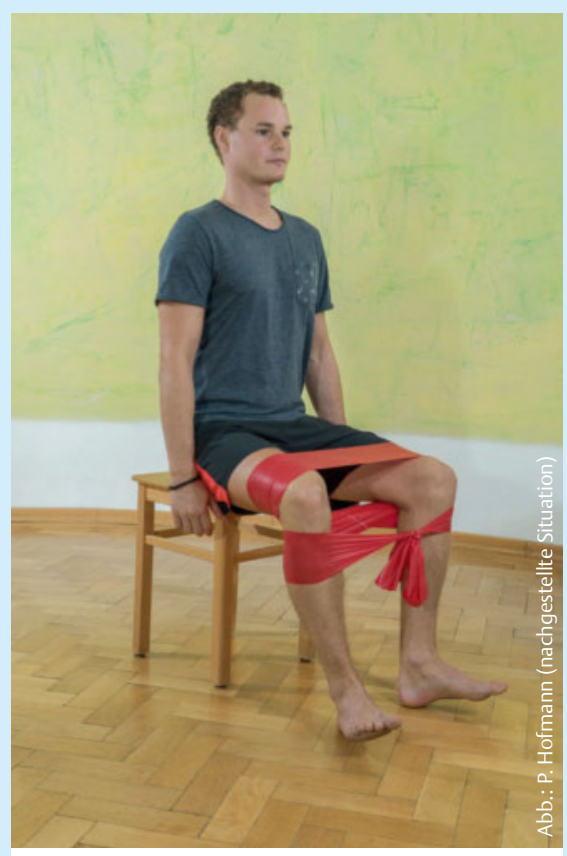

\section{Der Zappler}

Mit dieser Übung kräftigen Sie die Hüftgelenkaußenrotatoren, die als Stabilisatoren des Beckenbodens gelten. Legen Sie im Sitz das Theraband um Ihre Ober- und Unterschenkel, indem Sie es einmal hinter den Beinen kreuzen. Setzen Sie sich aufrecht hin. Heben Sie den Vorfuß und die Zehen an, sodass nur noch die Fersen Kontakt zum Boden haben. Ziehen Sie mit Ihren Knien das Theraband auseinander und wippen Sie am Endpunkt. Je nach Spannungsgrad des Therabandes wiederholen Sie die Übung 10 bis $20 \mathrm{Mal}$, zumindest aber bis Sie eine deutliche Ermüdung in der Hüftmuskulatur spüren.

Wichtig: Achten Sie darauf, dass Sie aufrecht sitzen bleiben und nicht zusammensacken.

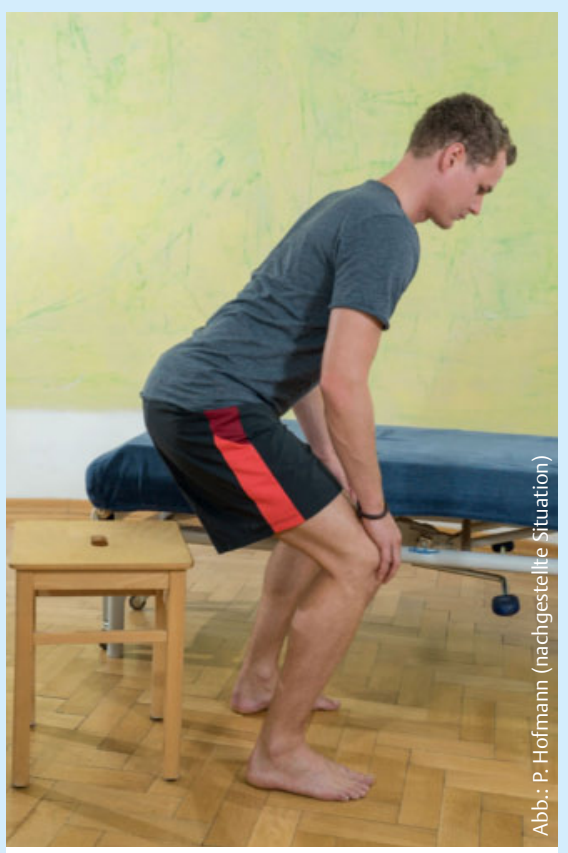

\section{Der Konzertpianist}

Um die Haltung zu trainieren, in welcher die Beckenbodenmuskulatur am besten arbeitet, neigen Sie Ihren Oberkörper im Sitz nach vorne. Stellen Sie sich vor, wie sich Ihr Steißbein im Raum nach hinten oben bewegt. Die Wirbelsäule bleibt stabil, der Bauch lang. Wenn sich Ihr Gesäß von der Unterlage löst, stehen Sie auf. Der Oberkörper bleibt nach vorne geneigt und das Steißbein strebt in der Vorstellung nach hinten oben auch beim Hinsetzen. Stellen Sie sich vor, Sie seien ein Konzertpianist, der seine Frackschwänze über den Hocker bringen möchte. Die Übung können Sie bei jedem Aufstehen und Hinsetzen durchführen.

Wichtig: Halten Sie Ihren Bauch lang und die Wirbelsäule stabil.

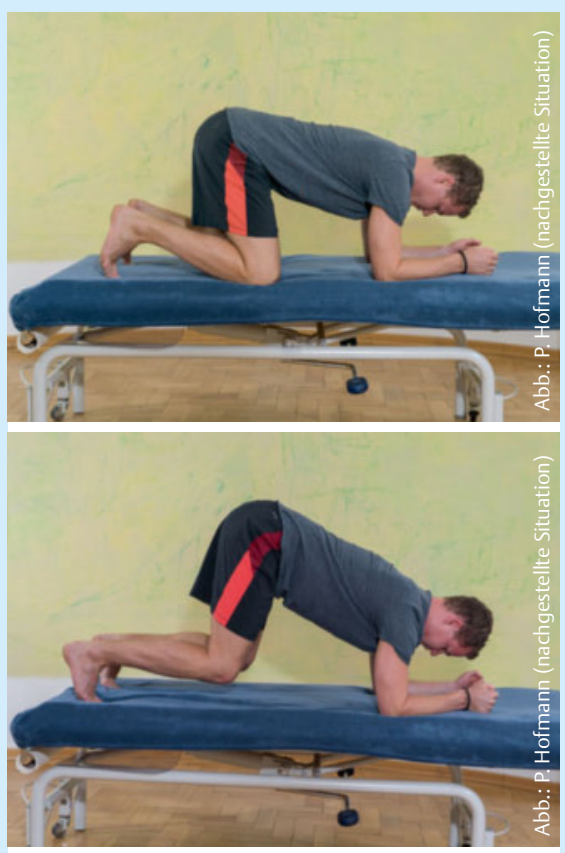

\section{Bankstellung}

Die Bankstellung eignet sich, um die Bauch- und Beckenbodenmuskulatur zu trainieren. Zudem erlernen Sie eine Rumpfspannung, bei der die untere Wirbelsäule weder abflacht noch durchhängt. Heben Sie im Unterarm- und Kniestütz beide Kniegelenke von der Unterlage ab - maximal $10 \mathrm{~cm}$. Halten Sie diese Position für 8 bis 10 Sekunden und machen Sie anschließend eine Pause von 2 Atemzügen. Beginnen Sie mit 2 Serien à 5 Wiederholungen und 1 Minute Pause und steigern Sie auf 3 Serien à 10 Wiederholungen.

Wichtig: Pressen Sie nicht, atmen Sie ruhig weiter. Wenn Sie kürzlich im Bauchraum operiert wurden, beginnen Sie mit der Übung erst 6 Wochen nach der Operation.
Wenn Sie Fragen zu den
Übungen haben, wenden
Sie sich gerne an uns: 

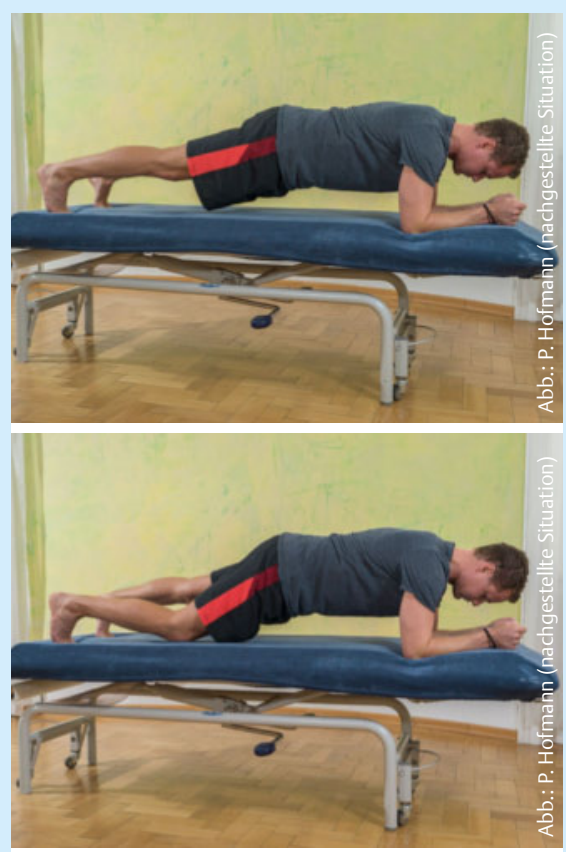

\section{Walking Planking}

Mit dieser Übung kräftigen Sie Ihre Rumpf-, Becken- und Beinmuskulatur. Des Weiteren trainieren Sie, Ihren Rücken gerade zu halten, ohne den Atemfluss zu stoppen. Gehen Sie in den Unterarm- und Zehenstütz, Ihr Becken und Ihre Kniegelenke sind von der Unterlage abgehoben - wie eine Planke. Bewegen Sie im Wechsel das rechte und das linke Kniegelenk jeweils dreimal hintereinander in Richtung Unterlage. Heben Sie dabei die Zehen nicht ab. Starten Sie mit $2 \times 5$ Wiederholungen mit 1 Minute Pause dazwischen. Steigern bis zu $3 \times 10$ Wiederholungen.

Wichtig: Führen Sie die Kniebewegungen aus, ohne das Becken mitzudrehen. Halten Sie die Luft nicht an, atmen Sie ruhig weiter. Den Blick richten Sie die ganze Zeit in Richtung Unterlage, ziehen Sie den Kopf nicht in den Nacken. Wenn Sie kürzlich im Bauchraum operiert wurden, beginnen Sie mit der Übung erst 6 Wochen nach der Operation.

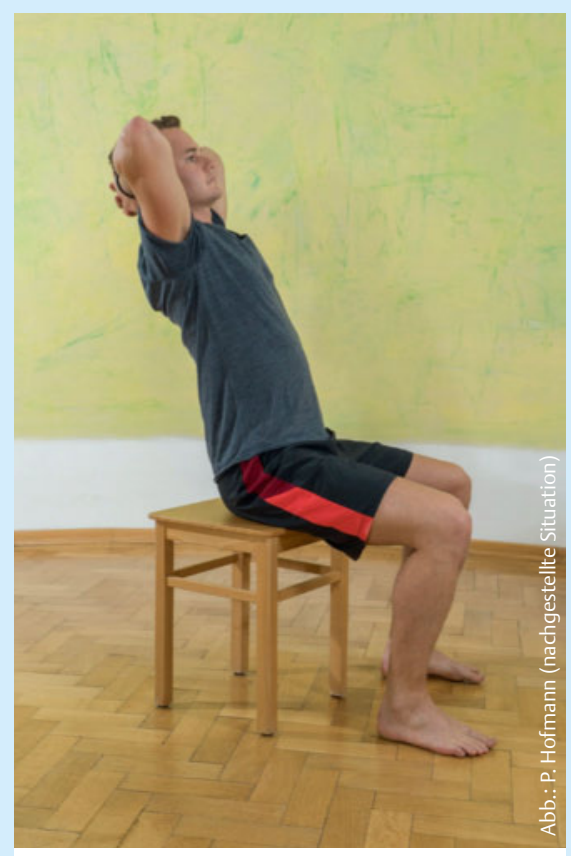

\section{Überall machbar}

Wenn Sie zwischendurch Ihre Bauchmuskulatur kräftigen und über den erhöhten Druck im Bauchraum Ihren Beckenboden aktivieren möchten, eignet sich diese Übung. Sie lernen zudem, im rückwärtigen Überhang die Streckung in der Wirbelsäule beizubehalten. Setzen Sie sich gerade hin und kippen Sie Ihr Becken nach vorne, sodass Sie vor den Sitzbeinhöckern sitzen. Rollen Sie das Becken nach hinten über die Sitzbeinhöcker, ohne die Streckung der Wirbelsäule zu verlieren. Die Bewegung findet ausschließlich in den Hüftgelenken statt. Halten Sie die Position 8 Sekunden lang und machen Sie anschließend eine Pause von 1 bis 2 Atemzügen. Wiederholen Sie die Übung so oft, bis Ihr Bauch kräftig zittert oder Sie Ihre Wirbelsäule nicht mehr gestreckt halten können.

Wichtig: Halten Sie Ihre Wirbelsäule stets stabil. Wenn Sie kürzlich im Bauchraum operiert wurden, beginnen Sie mit der Übung erst 6 Wochen nach der Operation.

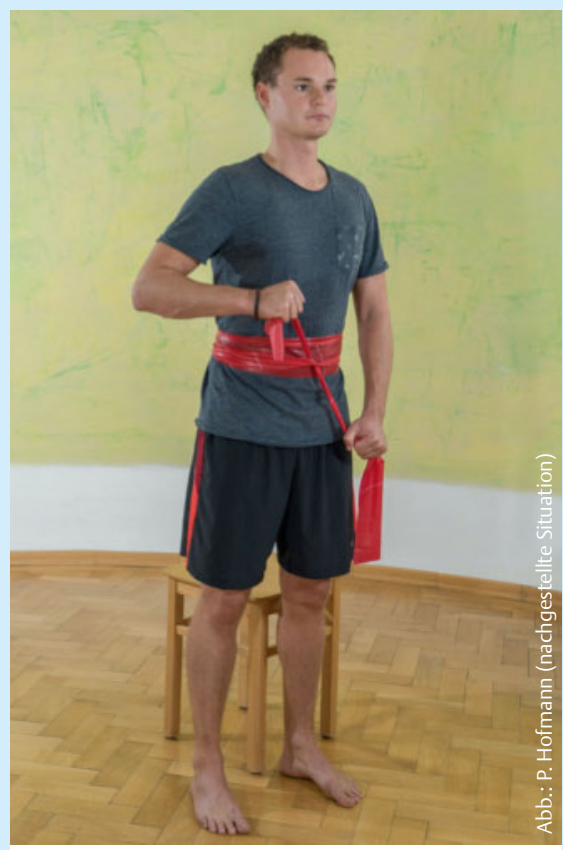

\section{Der Bauchbändiger}

Mit dem Bauchbändiger lernen Sie, Ihr Zwerchfell unter erschwerten Bedingungen einzusetzen. Die Übung kräftigt das Zwerchfell und reaktiviert den Beckenboden. Binden Sie sich ein Theraband (mindestens rot, besser blau) um den eingezogenen Bauch. Sie sollen weiterhin gut atmen und Ihre Atmung in die Bauchhöhle lenken können, ohne dass Sie verstärkt pressen müssen. Mit dieser Bauchbinde machen Sie nun Ihre Gymnastik oder Nordic Walking, gehen spazieren oder joggen. Anfangs genügen 5 bis 10 Minuten. Steigern Sie die Zeit peu à peu auf 20 bis 30 Minuten.

Wichtig: Verlagern Sie Ihren Atem nicht in den Brustbereich, behalten Sie bewusst die Bauchatmung bei. 